\title{
Context Classification in Energy Resource Management of Residential Buildings using Artificial Neural Network
}

\author{
Bruno Madureira, Tiago Pinto, Filipe Fernandes, Zita Vale, Carlos Ramos \\ GECAD - Research Group on Intelligent Engineering and Computing for Advanced Innovation and \\ Development, Institute of Engineering, Polytechnic of Porto, Portugal \\ \{brmad, tmcfp, fijgf, zav, csr\}@isep.ipp.pt
}

\begin{abstract}
This paper proposes an Artificial Neural Network (ANN) based approach to classify different contexts, with the goal of enhancing the management of residential energy resources. The increasing penetration of renewable based generation has completely changed the paradigm of the power and energy sector. The intermittent nature of these resources requires the system to incentivize the adaptability of consumers in order to guarantee the balance between generation and consumption. This leads to the emergence of several incentives with the objective of increasing the flexibility from the consumer's side. This, allied to the increasing price of electricity, leads to an increasing need from for consumers to adapt their consumption in order to improve energy efficiency, decrease energy bills, and achieve a better use of their own generation resources. With this, several House Management Systems (HMS), and Building Energy Management Systems(BEMS) have emerged. These systems allow adapting the consumption (or suggesting changes in consumers' habits) according to several factors. However, in order to make this management truly smart, there is a need for adaptation to different contexts, so that changes can be done accordingly to the different situations that are faced at each time. This paper addresses this problem by proposing a novel methodology that enables classifying different situations in different contexts, according to different contextual variables.
\end{abstract}

Index Terms - Artificial Intelligence, Artificial Neural Networks, Context Awareness, House Management Systems

\section{INTRODUCTION}

$\mathrm{C}$ ontext is any information that can be used to characterize the situation of an entity. An entity is a person, place, or object that is considered relevant to the interaction between a user and an application, including the user and the application themselves [1]. The development of a method that identifies and analyses contexts implies context awareness, i.e., the idea that artificial intelligence can respond to consumer needs based on information taken from the environment that surrounds him to provide relevant information or services to the consumer, where its relevance depends on the user's task. Although the notion of context

The present work has been developed under the EUREKA - ITEA2 Project M2MGrids (ITEA-13011), Project SIMOCE (ANI|P2020 17690), and has received funding from FEDER Funds through COMPETE program and from National Funds through FCT under the project UID/EEA/00760/2013. awareness has been around for several decades, there are still two major challenges in this issue: finding ways of collecting data that do not sacrifice consumer and the difficulty of removing relevant information from big data [2]. Context aware systems are especially relevant in dynamic and complex environments, such as the power and energy sector.

The consumption of electricity is increasing all around the world. For example, in the United States, for the year 2011, $22.00 \%$ of electricity consumption comes from residential consumers [3]. On the other hand, a report of Europe (EU-27) talks about the increase of global consumption in 2012. In this context, $29.60 \%$ of the total electricity is from residential consumers [4]. In the study of electricity consumption in Brazil for the year 2012, residential consumers represent $24.92 \%$ of total electricity consumption [5].

Consumers are active resources in the context of smart grids [6], thus the management systems must take into account the information of each equipment importance in the residential consumers. Management systems need to consider novel characteristics and advanced functions in response to the new context (electric vehicles penetration, interaction with external operators, among other). In several works these type of management system are defined as a smart home. A residential consumer with communication between all devices enabling the monitoring, control and remote access of the management system provides a smart home [7]. This type of systems should enable effectively managing consumption, storage, distributed generation and the participation in Demand Response (DR) events [8], [9].

The electricity consumption based on the interaction with an external entity can be reduced by the automatic participation in DR events of smart home. The communication between smart home and external entity is performed by smart meters. This smart equipment enables bidirectional communications with: monitoring in small time steps, information in real time about energy prices and costs and the remote control of the electricity demand management $[10,11]$. DR programs can represent an important energy resource but the industrial and large commerce consumers are the main focus to apply it. New developments show the DR events to get more flexible response applying the programs in small consumers as the residential consumers $[8,12]$.

Taking into account the needs identified in this field, the aim of this paper is to study and develop a method for 
analyzing, identifying and defining different contexts of resource usage in homes. A data-mining approach is used, namely a clustering method [13], which groups different historical events related to consumption measures in a home, the associated devices usage at each moment, and related contextual variables (e.g. temperature, humidity, external luminosity, etc). This clustering process allows identifying situations with similar characteristics, and thus enable the identification of different contexts of consumption and devices' usage. In this paper, a novel methodology based on the application of an Artificial Neural Network (ANN) [14] is proposed to classify new situations in different contexts, according to different available contextual variables. The proposed method is used to improve home resource management methods of the Supervisory control and data acquisition (SCADA) House Intelligent Management (SHIM) system [15], giving them ability to adapt to different contexts.

After this introduction, section II provides an overview on artificial neural networks. Section III presents the proposed methodology which is separated into two categories: context analysis and classification. Section IV presents the results of a case study using data that represents the energy consumption of a generic home during one year (2014) and features the measurements of several devices' consumption as well as of several contextual variables (e.g. season, temperature, number of residents inside the home). Finally, the most relevant conclusions are presented in section $\mathrm{V}$.

\section{RELATED WORK}

According to [16] a smart home should include three main elements: internal communication network, intelligent control systems, and home automation. Moreover, House Management Systems (HMS) should be able to effectively manage the consumption, distributed generation units, electric vehicles, and the participation in DR events, e.g. dynamic energy tariffs [17].

On the other hand, HMS should consider the consumption efficiency, the minimization of the energy bill, and the required comfort levels in the operation context [18]. Reference [19] proposes the use of user location systems, motion detection, and measurement/control devices [19], with the objective of determining users' behaviour profiles. In this way, some devices can be turned off e.g. when there is no one in a certain room. A HMS that joins the consumption management of electricity and gas is proposed in [20]. This work also addresses the active participation of users' no enable the enhancement of the system performance. The variables that impact the consumption are analysed in [21], such as comfort and the weather.

HMS solutions are emerging, despite the use of HMS is still not widespread. Reference [22] appoints some obstacles to the use of HMS, such as the lack of awareness of the existing technologies; prices of current solutions; and fragile user interfaces. These barriers, together with a deficient use of the available data in real time, are hurdles that must be addressed promptly. The development of algorithms that allow the computer to learn and improve its performance based on data (also known as machine learning) is, essential to surpass the identified gaps. In this sense, the tasks performed are normally divided into the following three categories: unsupervised, supervised and reinforcement learning.
Unlike unsupervised learning, supervised learning requires already pre-defined outputs [23]. Nowadays, the development of the field of research has been almost explosive. ANN's are at the forefront of computational systems designed to produce, or at least mimic, intelligent behavior [24]. In computer science, neural networks gained a lot of steam over the last few years in areas such forecasting, data analytics, as well as data mining [25]. There are many types of ANN such as: Feed Forward Neural Network, Self-Organizing Map (SOM), Hopfield Neural Network, Simple Recurrent Network (SRN), Simple Recurrent Network (SRN), Feed forward Radial Basis Function (RBF), among many others. Within these, the focus will be on Feed Forward Neural Network (FFNN) since it is the type of ANN used in the proposed methodology.

\section{PRoposed Methodology}

This chapter describes the methodology used to develop a method of analysis and definition of contexts with the application of the ANN classification model. The proposed method is divided into two categories: Context Analysis and Classification.

\section{A. Context Analysis}

The context analysis method is designed for analyzing, identifying and defining different contexts of resource usage in homes. For this, a data-mining approach is used, namely a clustering method [13], which groups different historical events related to consumption measures in a home, the associated devices usage at each moment, and related contextual variables (e.g. temperature, humidity, external luminosity, etc). This clustering process allows identifying situations with similar characteristics, and thus enable the identification of different contexts of consumption and devices' usage.

For the data mining process, a method of the unsupervised learning branch has been chosen, more precisely, a clustering method by iterative optimization (partitional clustering), the Kmeans clustering algorithm [13]. This clustering process seeks to organize a set of objects into $k$ clusters so that the inserted objects in a given cluster have high similarity level and a low level of similarity with objects belonging to different groups.

Given a set of observations $\left(x_{1}, x_{2}, \ldots, x_{n}\right)$ where $\mathrm{n}$ is the number of observations considered and in which each observation is a d -dimensional vector. The clustering process aims to perform the division of $\mathrm{n}$ observations into $\mathrm{k}$ clusters $\left(C_{1}, C_{2}, \ldots, C_{k}\right)$ to minimize the sum of square errors, WCSS (Within Cluster Sum of Squares).

$$
\min \sum_{i=1}^{k} \sum_{x \in c_{i}}\left\|x-\mu_{i}\right\|^{2}
$$

Where $\mu_{i}$ is the average points in $C_{i}$ and $C_{i}$ being the centroid of the cluster. With the aim of minimizing the equation (1), the iterative k-means clustering process consists of two steps:

- Association, each observation $x$ is linked to the cluster C whose mean value can WCSS minimum;

- Update the centroids, considering new observations 
associations, new means of each cluster values are calculated, given a new centroid $\mu_{\mathrm{i}}$.

The algorithm execution continues until the convergence process has finishes, in other words, when the combination of observations to different clusters no longer varies, finding a fixed point or local minima.

$\mathrm{K}$-means is a relatively efficient method of clustering but it requires to specify the number of considered clusters beforehand and the success or failure of the method will depend on this indication. Unfortunately, there is still no general procedure to find the correct number of clusters. However, there are some criteria to suggest an optimal $k$, e.g., the number of groups that allow the best grouping for the collected data. These criteria are mainly based on two aspects:

- Compaction: a group of measures that assess the compactness of the cluster based on variance. Measures how closely related objects in a cluster are;

- Separation: a group of measures that assess the distinctive as well or separate a cluster is from other clusters.

The following criteria are considered in this work: Calinski Harabasz (CH), Davies Bouldin (DB), Gap Value (GAP) and Silhouette (S).

\section{B. Classification}

The proposed classification model intends to enable identifying the context in which new observations or events should be associated to. The ANN used in this proposed method is a feedforward neural network. Feedforward networks consist of a series of layers. The first layer has a connection from the network input. Each subsequent layer has a connection from the previous layer. The final layer produces the network's output.

\section{1) ANN model}

The considered ANN is a Multi-Layer Perceptron (MLP) feedforward neural network, which considers the contextual information about the event in time. The output is the corresponding context. A study that supports this MLP topology is shown in [26]. Figure 1 depicts an overview of the MLP topology.

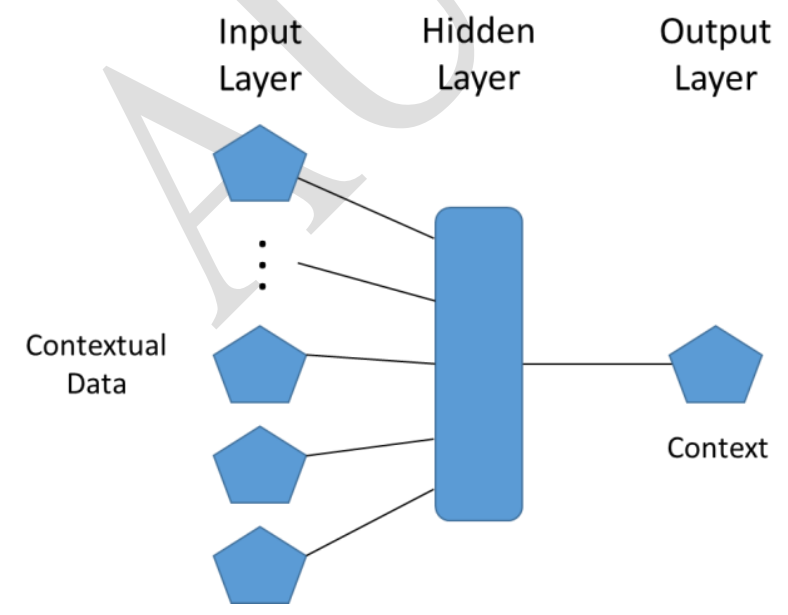

Fig. 1. Artificial Neural Network topology
The training algorithm is backpropagation using the gradient descent method [27]. The squared error function $E$ for the single output neuron is defined as in (2).

$$
E=\frac{1}{2}(t-y)^{2}
$$

where $t$ is the target output for a training sample, and $y$ is the actual output of the output neuron.

For each neuron $j$, its output $o_{j}$ is defined by feedforward calculation, as in (3).

$$
o_{j}=f\left(\sum_{k=1}^{n} w_{k j} x_{k}\right)
$$

where $n$ is the number of input units to neuron $j$, and $w_{k j}$ is the weight between neurons $k$ and $j$. The logistic function is used as activation function $f$, as in (4) [28].

$$
f(z)=\frac{1}{1+e^{-z}}
$$

\section{2) Assessment measures}

Mean absolute percentage error (MAPE) expresses the accuracy of the model by comparing the real vs. forecast values and can be defined by the equation (5):

$$
\text { MAPE }=\frac{1}{n} \sum_{t=1}^{n}\left|\frac{A_{t}-F_{t}}{A_{t}}\right|
$$

Where, ${ }^{A} t$ is the real value, $F_{t}$ is the forecast value and ${ }^{n}$ is the number of considered samples.

Class error (CE) expresses the accuracy of the forecast model by comparing the number of samples that wrongly predict the number of clusters with the total number of considered samples and can be represented by the equation (6):

$$
C E=\frac{n_{\text {error }}}{n}
$$

Where, $n_{\text {error }}$ is the number of samples with the wrong class, ${ }^{n}$ is the number of considered samples.

\section{EXPERIMENTAL FINDINGS}

This chapter is structured in two parts: (i)Specification, where the description of the procedure used to obtain the results is provided; and (ii) Results, where the outcomes of the study are showcased. Finally, a summary is also presented, including a global analysis of the achieved results.

\section{A. Specification}

The data used in this case study represents the energy consumption of a generic home during the year 2014 (64 samples) and features the measurements of the following variables: month, day of the week, hour, season, temperature, number of residents inside the home, total power usage, indication $\mathrm{ON} / \mathrm{OFF}$ of lighting by division (bedroom, living room, hall, kitchen) and consumption of devices (microwave, oven, coffee machine, fridge 1 , fridge 2 , washing machine, HVAC, water heater, room TV, living room TV). 
The set of 64 samples of 21 variables is decomposed into groups that represent different situations (or contexts). The considered contexts are of different natures, trying to gather information about different situations. Out of several scenarios that have been analyzed, this focuses on the experimental findings considering three scenarios that relate specific variables, namely: Temperature vs. HVAC, Hour vs. Power and Number of Residents vs. Power.

To classify new observations as being part of a specific context, the contextual data (described in the previous paragraph) is used to feed the ANN. The classification process utilizes two thirds of the sample values as inputs and the corresponding clustering results as targets. Being the remaining one third of data used as test values and the corresponding clustering results used as real values. This way it is possible to determine the performance of ANN by comparing the output classification values with the real values. To classify observations for different contexts, the proposed feedforward ANN changes only in the number of hidden layers and in the number of neurons inserted in these.

\section{B. Results}

For each scenario the four optimal $\mathrm{k}$ criteria exposed in section III A. are used, namely CH, DB, GAP and S. Each of these criteria results in a distinct number of optimal k, which results in a different number of considered contexts. The ANN is then used to classify the new observations as being part of one of the contexts. Results analysis consists in the comparison between the forecast classification values vs. the real value, visually and by using the MAPE and CE index results.

\section{1) Temperature vs. HVAC}

The purpose of this grouping is to find patterns in the usage of the HVAC system by residents depending on the temperature, the following variables are selected:

- Outside temperature

- Indication ON / OFF of the HVAC system

Figures 2, 3, 4 and 5 illustrate the classification performance by comparing the classification (forecast) result and the real context value for each test observation. The $\mathrm{xx}$ axis represents the several observations, and the yy axis represents the context number in which each observation is classified.

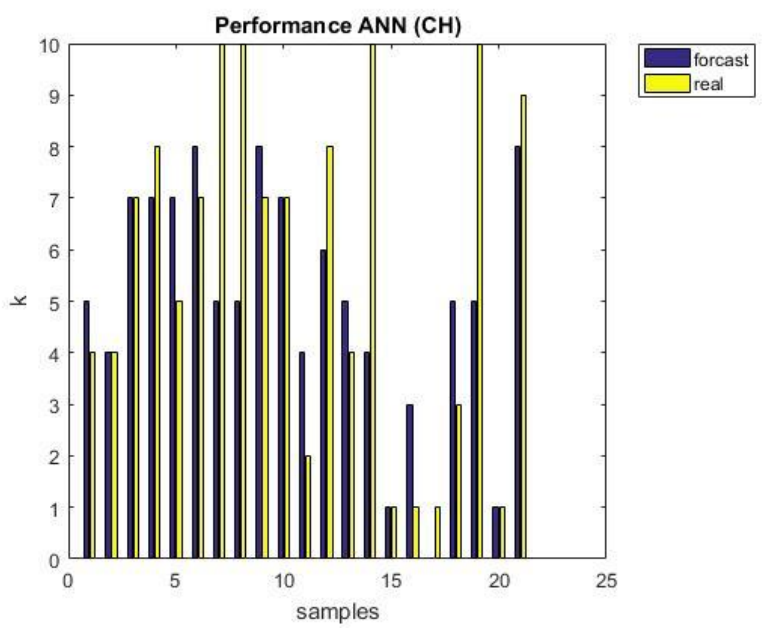

Fig. 2. Classification performance (Temperature vs. HVAC - CH criterion)
The MAPE value achieved in this case $(\mathrm{CH}$ criterion, which results in using 10 contexts) is $0,4018 \%$ and the CE value is 0,7619 .

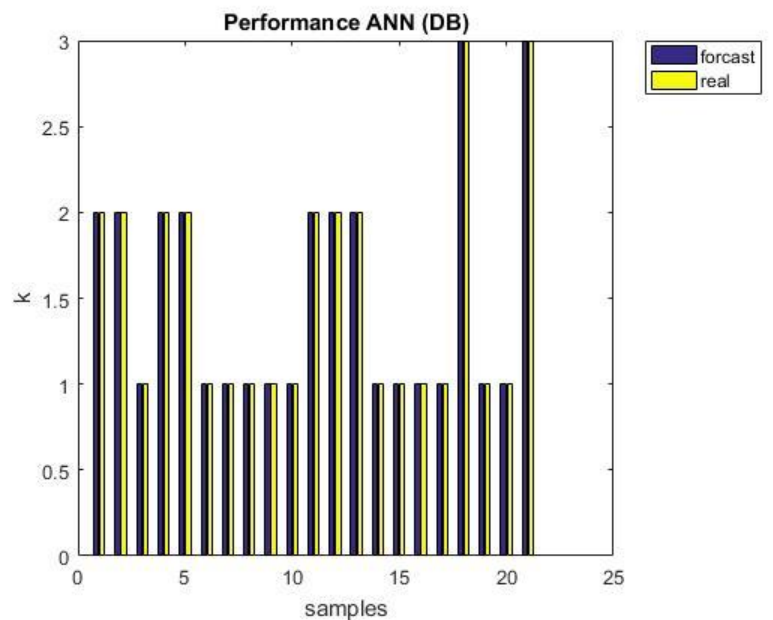

Fig. 3. Classification performance (Temperature vs. HVAC - DB criterion)

Using the BD criterion, the number of contexts is 3 , and the MAPE value obtained is $0 \%$ and the CE value is also 0 .

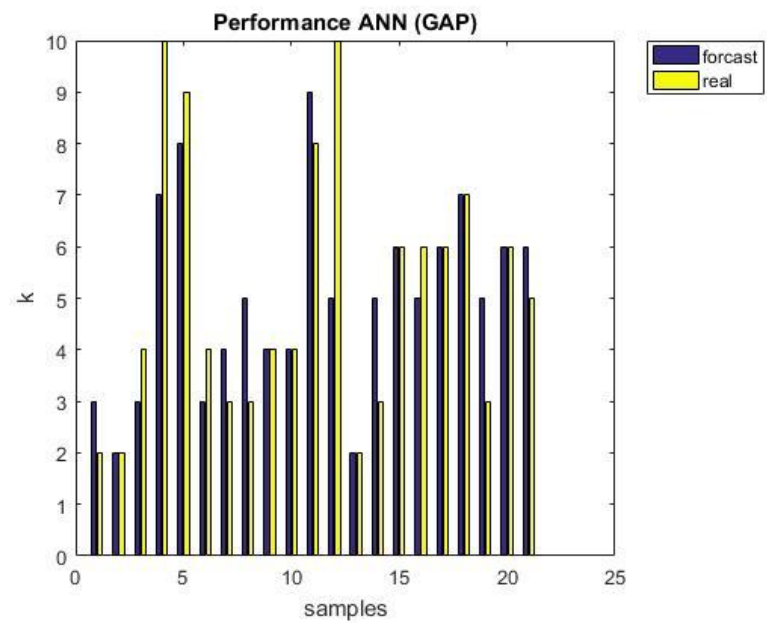

Fig. 4. Classification performance (Temperature vs. HVAC - GAP criterion)

Applying the GAP criterion results in 10 contexts; the MAPE value obtained is $0,2255 \%$ and the $C E$ value is 0,6190 .

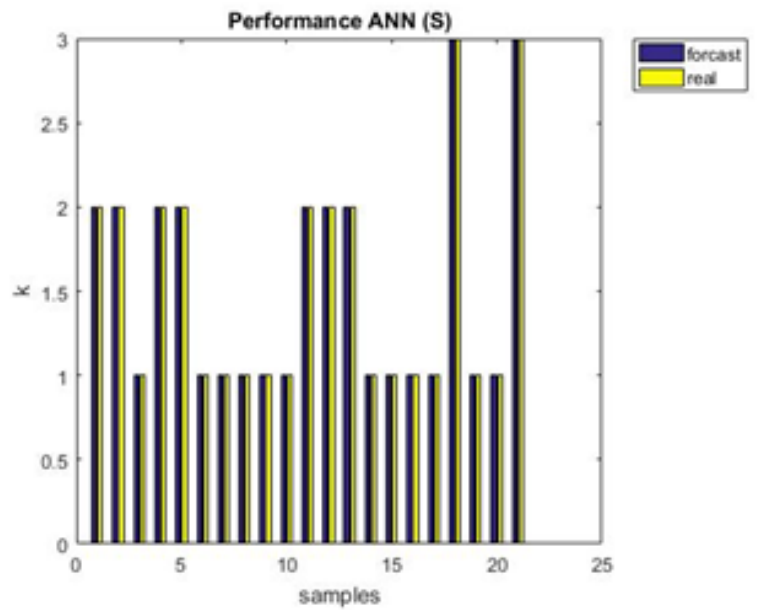

Fig. 5. Classification performance (Temperature vs. HVAC - S criterion) 
Using the $\mathrm{S}$ criterion results in 3 contexts and the achieved MAPE value for this case is $0 \%$ and the CE value is also 0 .

Table I presents the summary of the clustering results obtained when using the different optimal $\mathrm{k}$ criteria for this scenario. Namely the number of clusters (contexts) that result from the application of each of criterion.

TABLE I

NUMBER OF CLUSTERS COMPARISON - TEMPERATURE VS. HVAC

\begin{tabular}{cc}
\hline \hline Number of clusters & Criteria \\
\hline 10 & $C H$ \\
3 & $D B$ \\
10 & $G A P$ \\
3 & $S$ \\
\hline \hline
\end{tabular}

From these results it is visible that the criteria $\mathrm{CH}$ and GAP overestimated the optimal value of $\mathrm{k}$. Using only 3 contexts: resulting from DB and S, three groups (contexts) are identified, which refer to: use of the HVAC system at low temperatures, HVAC system OFF and use of the HVAC system at high temperatures.

Table II summarizes the classification errors obtained for the "Temperature vs. HVAC" scenario.

TABLE II

MAPE AND CE VALUES COMPARISON - TEMPERATURE VS. HVAC

\begin{tabular}{ccc}
\hline \hline MAPE $(\%)$ & CE & Criteria \\
\hline 0,4018 & 0,7619 & $C H$ \\
0 & 0 & $D B$ \\
0,2255 & 0,6190 & $G A P$ \\
0 & 0 & $S$ \\
\hline \hline
\end{tabular}

Analyzing the achieved results, it can be seen that the prediction models based on the DB and S criteria significantly outperform the other two criteria (GAP and $\mathrm{CH}$ ). So far, what was identified as the best clustering groupings have led to the best predictions. Using a large number of clusters/contexts leads to a poor classification performance.

\section{2) Hour vs. Power}

This scenario aims to find power usage patterns by relating the total power consumption with the hour of day. To this end, the following variables are selected:

- Hour of the day

- Total power consumption

Figures $6,7,8$ and 9 illustrate the classification performance by comparing the classification (forecast) result and the real context value for each test observation. The $\mathrm{xx}$ axis represents the several observations, and the yy axis represents the context number in which each observation is classified.

The MAPE value achieved in this case $(\mathrm{CH}$ criterion, which results in using 10 contexts) is $0,2985 \%$ and the CE value is 0,6667 .

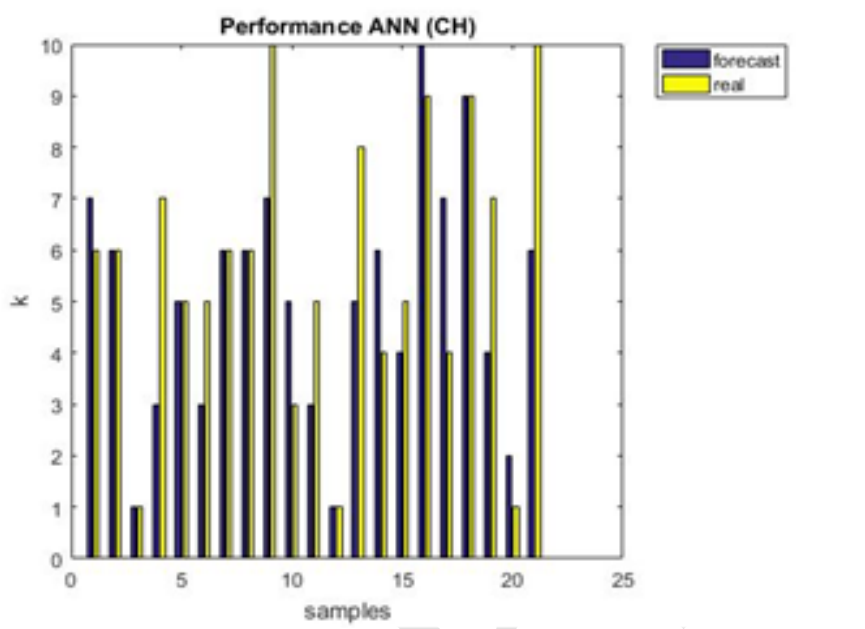

Fig. 6. Classification performance (Hour vs. Power - $\mathrm{CH}$ criterion)

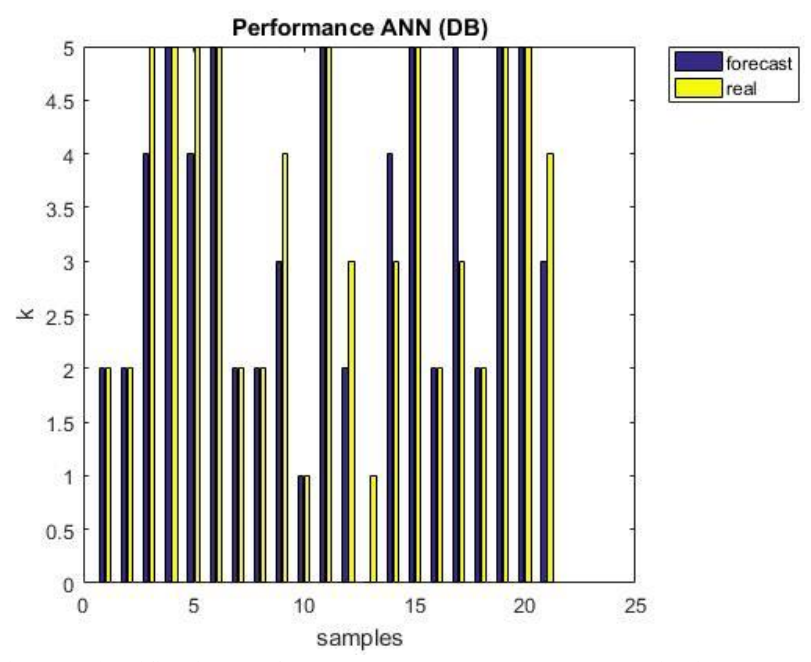

Fig. 7. Classification performance (Hour vs. Power - DB criterion)

Using the BD criterion, the number of contexts is 5 , and the MAPE value obtained is $0,1540 \%$ and the CE value is 0,3809 .

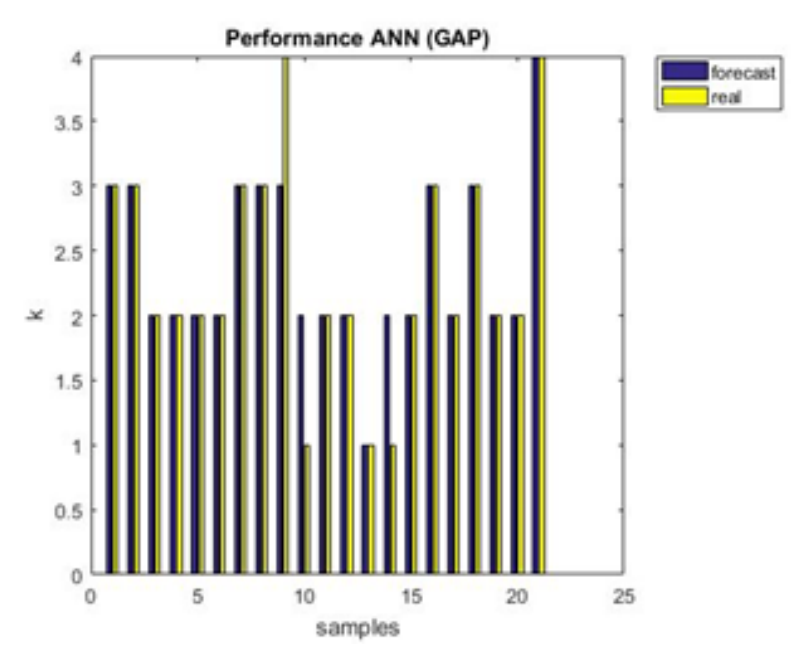

Fig. 8. Classification performance (Hour vs. Power - GAP criterion)

Applying the GAP criterion results in 4 contexts; the MAPE value obtained is $0,1071 \%$ and the CE value is 0,1428 . 


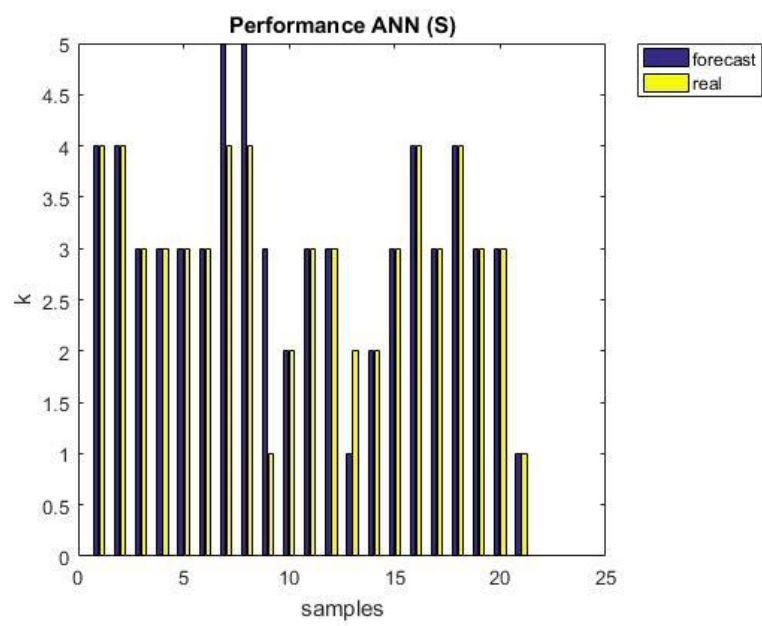

Fig. 9. Classification performance (Hour vs. Power - $\mathrm{S}$ criterion)

Using the $\mathrm{S}$ criterion results in 5 contexts and the achieved MAPE value for this case is $0,1071 \%$ and the $\mathrm{CE}$ value is 0,1905 .

Table III presents the summary of the clustering results obtained when using the different optimal $\mathrm{k}$ criteria for this scenario. Namely the number of clusters (contexts) that result from the application of each of criterion.

TABLE III

NUMBER OF CLUSTERS COMPARISON - HOUR VS. POWER

\begin{tabular}{cc}
\hline \hline Number of clusters & Criteria \\
\hline 10 & $C H$ \\
5 & $D B$ \\
4 & $G A P$ \\
4 & $S$ \\
\hline \hline
\end{tabular}

From these results it is visible that the criterion $\mathrm{CH}$ overestimated the optimal value $\mathrm{k}$. Being also identified four or five groups that could be labeled as: morning, noon, evening and night or dawn, morning, noon, afternoon and night. The main difference is that the DB criterion separates morning into two separate groups while the other two criteria (GAP and S) do not.

Table IV summarizes the classification errors obtained in this paper for the "Hour vs. Power" scenario.

TABLE IV

MAPE AND CE VALUES COMPARISON - HOUR VS. POWER

\begin{tabular}{ccc}
\hline \hline MAPE $(\%)$ & CE & Criteria \\
\hline 0,2985 & 0,6667 & $C H$ \\
0,1429 & 0,3809 & $D B$ \\
0,1540 & 0,1428 & $G A P$ \\
0,1071 & 0,1905 & $S$ \\
\hline \hline
\end{tabular}

Analyzing the results obtained, it can be said that the criteria GAP and S significantly outperform the other prediction models. In this case, there was doubt between four or five clusters being the best clustering grouping. If we go by these results presented, it can be said that the $S$ criterion provided the best MAPE error and that the criteria GAP provided the best $\mathrm{CE}$ error.
The DB criterion, which identified five groups as the best optimal k, despite giving an acceptable MAPE error, the CE error is a lot bigger when compared with the previous two criteria mentioned. So it can be said that an optimal $\mathrm{k}$ of four groups (contexts) for this scenario leads to better predictions and is possibly the best option.

\section{3) Number of Residents vs. Power}

This grouping aims to find usage patterns by relating the total power consumption with the number of people in the residence at a given time. In order to achieve this goal, the following variables were selected:

- Number of residents

- Total power consumption

Figures 10, 11, 12 and 13 illustrate the classification performance by comparing the classification (forecast) result and the real context value for each test observation. The $\mathrm{xx}$ axis represents the several observations, and the yy axis represents the context number in which each observation is classified.

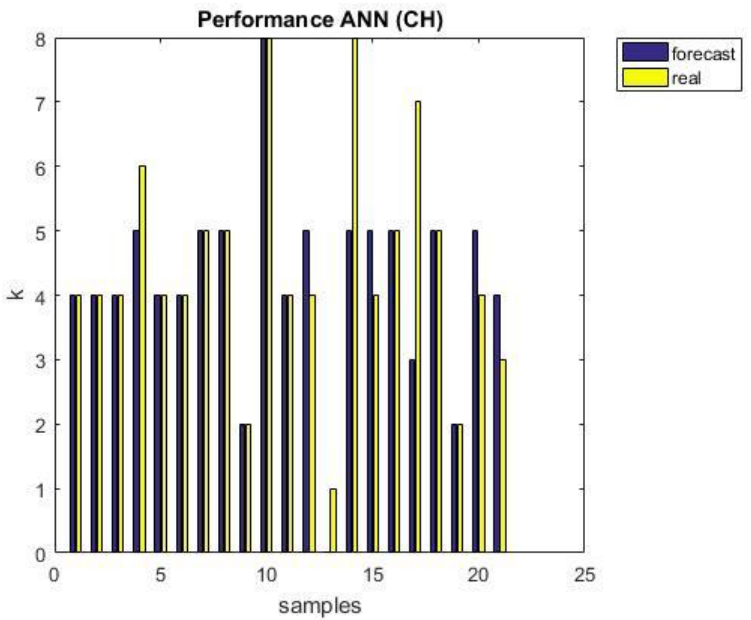

Fig. 10. Classification performance (Num. of Residents vs. Power - $\mathrm{CH}$ criterion)

The achieved MAPE value ( $\mathrm{CH}$ criterion, which results in using 8 contexts) is $0,1522 \%$ and the $\mathrm{CE}$ value is 0,3809 .

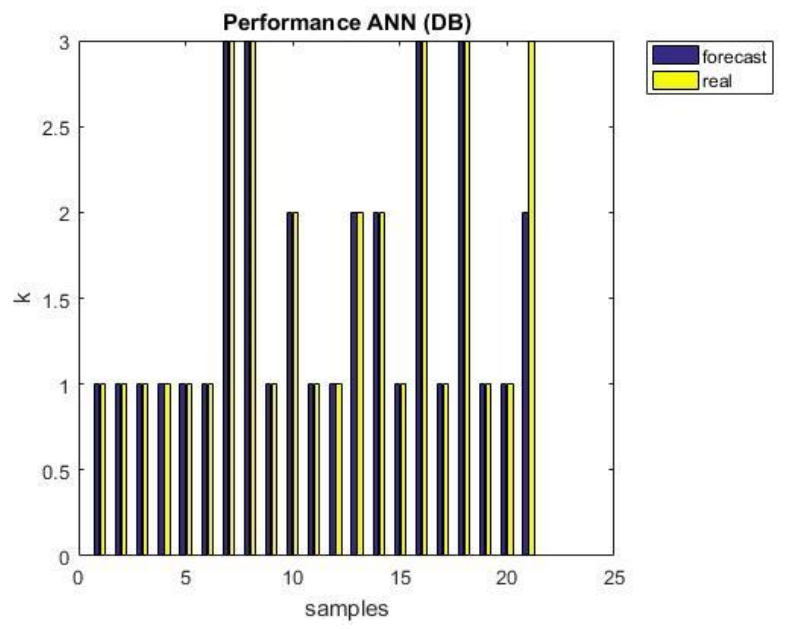

Fig. 11. Classification performance (Num. of Residents vs. Power - DB criterion) 
Using the $\mathrm{BD}$ criterion, the number of contexts is 3 , and the MAPE value obtained is $0,0159 \%$ and the $C E$ value is 0,0476

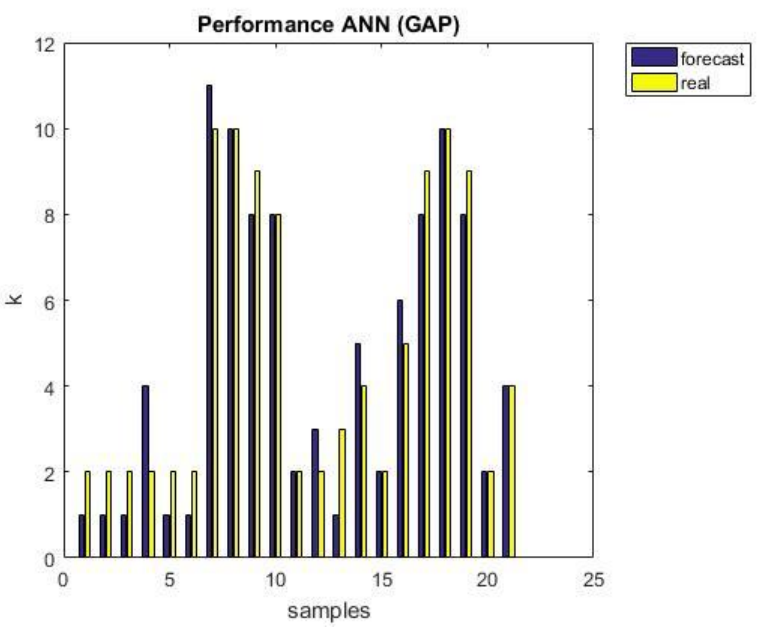

Fig. 12. Classification (Num. of Residents vs. Power - GAP criterion)

Applying the GAP criterion results in 10 contexts; the MAPE value obtained is $0,2643 \%$ and the $C E$ value is 0,6667

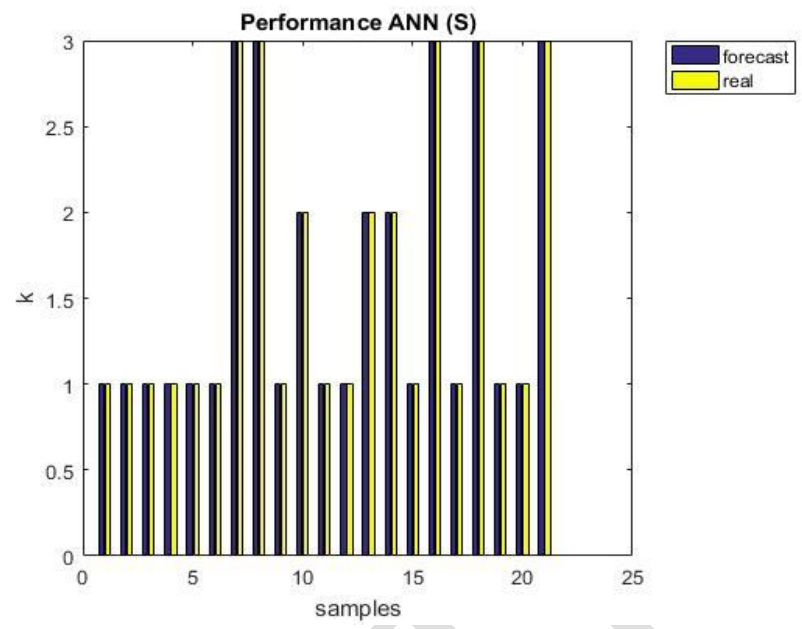

Fig. 13. Classification (Num. of Residents vs. Power - GAP criterion)

Using the $\mathrm{S}$ criterion results in 3 contexts and the achieved MAPE value for this case is $0 \%$ and the CE value is also 0 .

Table $\mathrm{V}$ presents the summary of the clustering results obtained when using the different optimal $\mathrm{k}$ criteria for this scenario. Namely the number of clusters (contexts) that result from the application of each of criterion.

TABLE V

NUMBER OF CLUSTERS COMPARISON - NUMBER OF RESIDENTS VS. POWER

\begin{tabular}{cc}
\hline \hline Number of clusters & Criteria \\
\hline 8 & $C H$ \\
3 & $D B$ \\
10 & $G A P$ \\
3 & $S$
\end{tabular}

From these results it is visible that $\mathrm{CH}$ and GAP criteria overestimated again the value of optimal $\mathrm{k}$. DB and S have identified three groups that could be labeled as: minimum capacity, average capacity and maximum capacity.
Table VI summarizes the classification errors obtained in this paper for the "Number of Residents vs. Power" scenario.

TABLE VI

MAPE AND CE VALUES COMPARISON - NUMBER OF RESIDENTS VS. POWER

\begin{tabular}{ccc}
\hline \hline MAPE $(\%)$ & CE & Criteria \\
\hline 0,1522 & 0,3809 & $C H$ \\
0,0159 & 0,0476 & $D B$ \\
0,2643 & 0,6667 & $G A P$ \\
0 & 0 & $S$ \\
\hline \hline
\end{tabular}

Analyzing the achieved results, similarly to the first scenario, it can be said again that the criteria DB and S significantly outperform the two criteria (GAP and $\mathrm{CH}$ ). In fact, using the context separation using the $\mathrm{S}$ criterion, the classification error is 0 under both error measures.

\section{Summary}

In this section, a brief analysis of the achieved results is performed, considering the studied scenarios.

Table VII presents the overview of the classification errors achieved with MAPE for all context scenarios and all clustering optimal $\mathrm{k}$ criteria.

TABLE VII

SUMMARY OF MAPE RESULTS IN ALL SCENARIOS AND OPTIMAL K CRITERIA

\begin{tabular}{|l|c|c|c|c|}
\hline & $\begin{array}{c}\text { Temperature } \\
\text { vs. HVAC }\end{array}$ & Hour vs. Power & $\begin{array}{c}\text { Num. Residents } \\
\text { vs. Power }\end{array}$ & Total \\
\hline CH & $40,18 \%$ & $29,85 \%$ & $15,22 \%$ & $28,42 \%$ \\
\hline DB & $0 \%$ & $15,40 \%$ & $1,59 \%$ & $5,66 \%$ \\
\hline Gap & $22,55 \%$ & $10,71 \%$ & $26,43 \%$ & $19,89 \%$ \\
\hline S & $0 \%$ & $14,29 \%$ & $0 \%$ & $4,76 \%$ \\
\hline
\end{tabular}

From Table VII It can be concluded that the best classification errors are obtained with the number of clusters that has been considered as ideal in the context definition (clustering) phase, i.e. for a lower number of clusters/contexts. It is also seen that for clustering with high number of clusters, the performance of ANN is significantly worse. DB and S criteria have proposed a smaller number of clusters/contexts in all scenarios, and this has resulted in a much better classification quality than when using the number of contexts provided by $\mathrm{CH}$ and GAP. Table VIII presents the overview of the classification errors achieved with $\mathrm{CE}$ for all context scenarios and all clustering optimal $\mathrm{k}$ criteria.

TABLE VIII

SUMMARY OF CE RESULTS IN ALL SCENARIOS AND OPTIMAL K CRITERIA

\begin{tabular}{|l|c|c|c|c|}
\hline & $\begin{array}{c}\text { Temperature } \\
\text { vs. HVAC }\end{array}$ & Hour vs. Power & $\begin{array}{c}\text { Num. Residents } \\
\text { vs. Power }\end{array}$ & Total \\
\hline CH & 76,19 & $66,67 \%$ & $38,09 \%$ & $60,31 \%$ \\
\hline DB & $0 \%$ & $35,09 \%$ & $4,76 \%$ & $14,28 \%$ \\
\hline Gap & $61,90 \%$ & $14,28 \%$ & $66,66 \%$ & $47,61 \%$ \\
\hline S & $0 \%$ & $19,05 \%$ & $0 \%$ & $5,35 \%$ \\
\hline
\end{tabular}

From Table VIII one can see that the forecast models based on the $\mathrm{S}$ criterion produced the best results $(4,76 \%$ and $5,35 \%)$ for MAPE and CE respectively, followed by the DB criteria $(5,66 \%$ and $14,28 \%)$. The other two criteria performed poorly, but the GAP criterion $(19,89 \%$ and $47,61 \%)$ was better than the CH criterion $(28,42 \%$ and $60,31 \%)$, mostly because of the "Hour vs. Power" scenario where successfully identified four groups as the optimal $\mathrm{k}$. 


\section{CONCLUSIONS}

The increasing penetration of renewable based generation of intermittent nature has brought a novel share of uncertainty into the power and energy sector. This, allied with the growing scarcity of fossil fuels leads to the increase of energy bills. Dealing with this new paradigm requires a larger dynamism and adaptability capability from consumers (in order to be able to change the consumptions patterns to make the best use of their own generated energy and to avoid hours of peak prices for consumption). In this scope, several HMS have emerged, which are able to deal with different variables and suggest the user some change of habits towards the minimization of the energy bill without compromising their comfort. However, these HMS still lack the capability of automatic adaptiveness to different contexts.

It is to overcome this gap that this work emerges, by proposing a novel methodology to automatically analyze and define different contexts for residential energy management. A clustering approach is used to group different situations into similar contexts. An ANN based classification approach is proposed to classify new observations/events as part as one of the pre-identified contexts.

Results show that the best classification results are achieved when considering the number of contexts that has been found ideal in the clustering phase (i.e. a small number of groups/clusters/contexts). Using a large number of contexts leads to the definition of several contexts with very small differences from each other, which harms the classification process (the classification method has difficulties in realizing in which context the new observation should fit), and it also does not improve the quality of the decision support, since a large number of contexts implies a larger difficulty in learning for each specific case and a larger difficult in understanding what each context means. Thus, using a smaller number of well-defined contexts is the ideal approach to be taken in order to include this type of context awareness in HMS.

\section{REFERENCES}

[1] Dey, Abowd, A conceptual framework and a toolkit for supporting the rapid prototyping of context-aware applications. 2001.

[2] Sama, M. et al., "Context-Aware Adaptive Applications: Fault Patterns and Their Automated Identification", IEEE Transactions on Software Engineering, vol. 36, no. 5, pp. 644-661, 2010

[3] J. Conti, J. Bearmon, and S. Napolitano, “Annual Energy Outlook 2013 with Projections to 2040," U.S. Energy Inf. Adm., vol. 383, pp. 1-233, 2013.

[4] European Energy Agency, "Final energy consumption by sector and fuel," Eur. Energy Agency, 2015.

[5] D. S. Gastaldello, A. N. de Souza, H. L. M. do Amaral, P. Faria, and Z. Vale, "Study of load curves concerning the influence of socioeconomic and cultural issues," IEEE PES Innovative Smart Grid Technologies, Europe. pp. 1-6, 2014.

[6] J. Z. Rokach, "Smart Houses in a World of Smart Grids," Electr. J., vol. 25, no. 3, pp. 94-97, 2012.

[7] Young-Min Wi, Jong-Uk Lee, and Sung-Kwan Joo, "Electric vehicle charging method for smart homes/buildings with a photovoltaic system," IEEE Trans. Consum. Electron., vol. 59, no. 2, pp. 323-328, May 2013.

[8] P. Faria and Z. Vale, "Demand response in electrical energy supply: An optimal real time pricing approach," Energy, vol. 36, no. 8, pp. 53745384, 2011.

[9] D. Tenfen, F. A. B. Lemos, R. C. Fernandes, and I. C. Decker, "Microgrids and Microgeneration in Brazilian Energy Market: a
Discussion of Regulatory and Commercial Aspects," in 9th LatinAmerican Congress on Electricity Generation and Transmission CLAGTEE, 2013.

[10] A. F. A. Aziz, S. N. Khalid, M. W. Mustafa, H. Shareef, and G. Aliyu, "Artificial intelligent meter development based on advanced metering infrastructure technology," Renew. Sustain. Energy Rev., vol. 27, pp. 191-197, 2013.

[11] S.-M. Jung, T.-K. Kim, H.-S. Seo, S.-J. Lee, and J. Kwak, "The prediction of network efficiency in the smart grid," Electron. Commer. Res., vol. 13, no. 3, pp. 347-356, 2013.

[12] Q. Liu, G. Cooper, N. Linge, H. Takruri, and R. Sowden, "DEHEMS: creating a digital environment for large-scale energy management at homes," IEEE Trans. Consum. Electron., vol. 59, no. 1, pp. 62-69, Feb. 2013.

[13] Anil K. Jain, Data clustering: 50 years beyond K-means. Pattern Recognition Letters. 2010.

[14] Hao, Q., Srinivasan, D. and Khosravi, A., "Short-Term Load and Wind Power Forecasting Using Neural Network-Based Prediction Intervals," IEEE Trans. on Neural Netw. and Learn. Syst., vol. 25, no. 2, pp. 303315, Feb. 2014

[15] F. Fernandes, H. Morais, P. Faria, Z. Vale, C. Ramos, SCADA house intelligent man-agement for energy efficiency analysis in domestic consumers, in: 2013 IEEEPES Conference on Innovative Smart Grid Technologies (ISGT Latin America). 2013.

[16] Li Jiang, Da-you Liu, and Bo Yang, "Smart home research," in Proceedings of 2004 International Conference on Machine Learning and Cybernetics (IEEE Cat. No.04EX826), 2004, vol. 2, pp. 659-663.

[17] Y. Fei and B. Jiang, "Dynamic Residential Demand Response and Distributed Generation Management in Smart Microgrid with Hierarchical Agents," Energy Procedia, vol. 12. pp. 76-90, 2011.

[18] Filipe Fernandes, Hugo Morais, Zita Vale, Carlos Ramos, "Dynamic load management in a smart home to participate in demand response events", Energy and Buildings, vol. 82, pp. 592-606, 2014

[19] M. Lee, Y. Uhm, Y. Kim, G. Kim, and S. Park, "Intelligent power management device with middleware based living pattern learning for power reduction," IEEE Trans. Consum. Electron., vol. 55, no. 4, pp. 2081-2089, Nov. 2009.

[20] Q. Liu, G. Cooper, N. Linge, H. Takruri, and R. Sowden, "DEHEMS: creating a digital environment for large-scale energy management at homes," IEEE Trans. Consum. Electron., vol. 59, no. 1, pp. 62-69, Feb. 2013.

[21] Y. Si, J. T. Kim, I. Y. Choi, and S. H. Cho, "Energy consumption characteristics of high-rise apartment buildings according to building shape and mixed-use development," Energy Build., vol. 46, pp. 123131,2012

[22] R. J. Meyers, E. D. Williams, and H. S. Matthews, "Scoping the potential of monitoring and control technologies to reduce energy use in homes," Energy Build., vol. 42, no. 5, pp. 563-569, 2010.

[23] Rudd, K., Di Muro, G. and Ferrari, S., "A Constrained Backpropagation Approach for the Adaptive Solution of Partial Differential Equations," IEEE Trans. on Neural Networks and Learning Systems, vol. 25, no. 3, pp. 571,584, Mar. 2014.

[24] Carlos Gershenson, Artificial Neural Networks for Beginners. 2003.

[25] DHT Technologies Inc., An Introduction to Artificial Neural Networks (ANN) - Methods, Abstraction, and Usage. "Introdution". [Online]. Available: http://www.dhtusa.com/media/NeuralNetworkIntro.pdf

[26] Pinto, T., Sousa, T.M., Vale, Z., "Dynamic Artificial Neural Network for Electricity Market Prices Forecast", IEEE 16th International Conference on Intelligent Engineering Systems (INES 2012), Costa de Caparica, Portugal, 13-15 June, 2012

[27] Pinto, T., et al "Adaptive Portfolio Optimization for Multiple Electricity Markets Participation" IEEE Transactions on Neural Networks and Learning Systems 27(8): 1720-1733 (2016)

[28] Rumelhart, D. E; Hinton G. E; Williams, R. J., "Learning representations of back- propagating error" London: Nature, v. 323, pp. 533-536, 1986 